\title{
Ingredient Scanner using Visa Tool
}

\author{
S. Lakshmi Harish, Dhanasekaran, Radhika Baskar
}

\begin{abstract}
It is an Application which is to gauge the calorie of nourishment, which was shifted relying upon its fixings and capacity in each cookery time and it is mandatory to ascertain calories of sustenance earlier it gets expended. In light of nourishment information, fixings that are parts of sustenance surely have various calories. This rag suggests a technique for fixing created nourishment admission guess utilizing sustenance learning and warm information. In this strategy, a duplicate of the sustenance is first perceived as the kind of nourishment, and afterward, the elements of the realized sustenance are recovered from the database with their nourishment information. At the same time, the picture is then sectioned into limits of fixing applicants, and every one of those limits is then characterized into fixings utilizing LabVIEW dependent on the fixings level which is fitting for each age classifications. The ordered fixings from all limits are at last determined for all-out calories dependent on zone proportion and sustenance information. The presentation of our proposed technique indicates satisfactory outcomes contrasting and the calories set up by the regular ruinous strategy.
\end{abstract}

\section{KEYWORDS: LABVIEW.}

\section{INTRODUCTION}

As indicated by the World Health Organization (WHO), -health advancement is that the strategy for sanctionative people to broaden the executives over, and to advance, their wellbeing. It proceeds onward the far aside from a focus on individual conduct towards an enormous shift of social and ecological intervention ( UN organization, 2014 ). Further, the Centers for ailment the board and deterrent (CDC) express that wellbeing advancing includes -creating, correspondence and conveying eudaimonia information and mediations abuse client centered and science-based methods to defend and progress the métier of different populationsll. Habitats for sickness the board and obstacle (2011). Wellbeing business sector portions may give esteemed signs concerning how social insurance associations may all the more explicitly target and introductory items and administrations for medicinal services client. Advances in the electronic medicinal record and in systematic capacities in wellbeing frameworks have prompted the usage of AI calculations and factual models to recognize hidden examples and collect significant understanding from promptly accessible patient information.

\footnotetext{
Revised Manuscript Received on September 10, 2019.

S. Lakshmi Harish, UG Scholar, Saveetha Institute of Medical and Technical Sciences, Chennai, Tamilnadu, India.

Dr. Dhanasekaran, Professor, Saveetha Institute of Medical and Technical Sciences, Chennai, Tamilnadu, India.

Dr. Radhika Baskar, Associate Professor, Saveetha Institute of Medical and Technical Sciences, Chennai, Tamilnadu, India.
}

This paper exhibits the value of exploitation data investigation in a very consideration setting, though filling in as an instructional exercise to direct scientists inside the space of wellbeing examination on an approach to incorporate each unsupervised and administered AI courses in an extremely solid structure. Furthermore, to serving to improve wellbeing advancement by influence tolerant data and AI for wellbeing market division, the outcomes give a letter of welcome for ensuing gifted frameworks applications in social insurance. In sub-period of rising human services costs and interest for administrations, the work of gifted frameworks holds the possibility to lift redid medicinal services by allowing consideration providers to speedily comprehend and focus in danger or at advantage wellbeing business sector fragments all together those wellbeing advancement ways might be custom-made viably to totally affect tolerant wellbeing results.

\section{STANDARDIZED IDENTIFICATION}

A standardized identification (likewise scanner tag) is a visual, machine-discernible image of the information, which means the information, as a rule, characterizes something about the article that moves the scanner tag. Normal scanner tags consistently speak to information by changing the lengths, breadths and dispersing's of comparable lines, and may well be alluded to as straight or one-D (1D). Progressed, double D (2D) decisions were settled, by methods for square shapes, spots, hexagons, and other symmetrical structures, scientific plans called lattice projects or $2 \mathrm{D}$ uniform empathies, in spite of the actual fact that they do not exploit bars thusly. Right off the bat, uniform empathies were just plaid by amazing optical scanners called scanner tag pursuers.

\section{PROPOSED SYSTEM}

This rag offers a technique of constituent-based food intake estimation mistreatment nutrition information and thermal knowledge. During this technique, a replica of the food is initial recognized as a sort of food, and parts of the recognized food area unit retrieved from the info with their nutrition knowledge. at the same time, the image is then divided into boundaries of ingredient candidates, and every one boundaries area unit then classified into constituents mistreatment using Lab VIEW based on the constituents level which is advisable for each age categories. 


\section{YIELD DESIGN}

Excellence yield stands that the unique, that encounters the necessities of the tip client then grants the learning unmistakably and appropriately. In any framework, aftereffects of taking care of are imparted to the clients and to the diverse framework through its creations. In vintage style it's controlled by any way the information is to be uprooted for swift requirement and conjointly the content yield. It's the chief vital and direct supply information to the client. Efficient and canny yield style improves the outline's association to aid user result-production.

\section{Architecture}

In IT, especially PCs and extra ongoing matrices, the style could be a term connected to each the technique and furthermore the result of thoroughly considering and expressing the structure, legitimate constituents, and furthermore the consistent interrelationships of a focal handling unit, its product framework, a system, or elective point in time. Partner configuration is a direct model, similar to the Open Systems Interconnection (OSI) direction model, foreseen as a model for explicit item structures or it is a specific item design, similar to that for partner Intel Pentium microchip or for IBM's OS/390 operational framework. Pc configuration is partitioned into 5 fundamental modules: input/yield, stockpiling, specialized, gadget, and procedure. In apply, all of those components (at times known as subsystems) is regularly previously mentioned to have partner plan, in this way, of course, setting adds to use and which implies. Interestingly, the term style means feeling that has less option than plan. The partner configuration could be a style, be that as it may, most styles don't appear to style. One module or a fresh out of the plastic new perform highlights a style that should coordinate among the style. An undifferentiated from term, plot, is thought of in light of the fact that the auxiliary a piece of the partner style.

\section{Example of handling events:}

When addressing tool communication, it's quite common for the instrument to need service from the checker once the checker isn't really staring at the theme. A tool will inform the controller via a service request (SRQ), interject, or a proof. Every of those is an asynchronous event, or just an occurrence. In VISA, you'll handle these and different events through either callbacks or a package row.

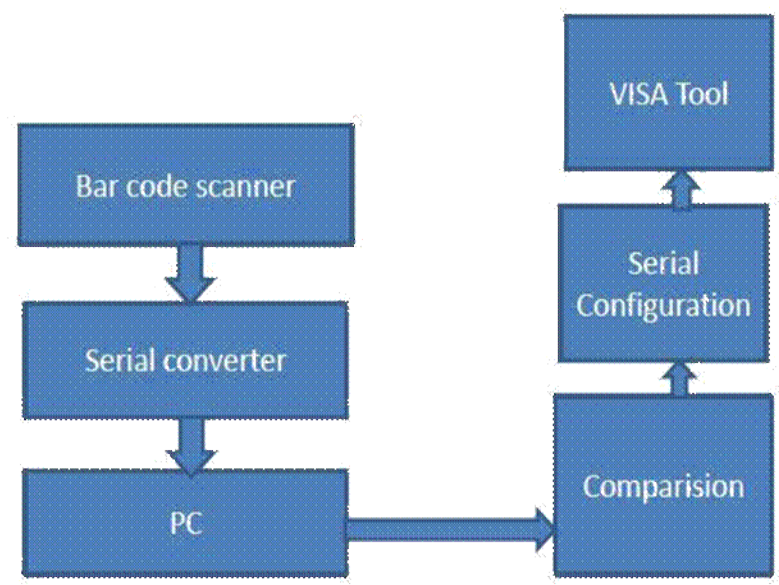

Architecture of the project
In this procedure, an image of the sustenance is first perceived as a kind of nourishment, and elements of the recognized sustenance and their sustenance information and example of brilliance and warm photographs. Some item unmistakably reflects their reasonableness to the client, while various others don't portray their appropriateness to the client. Reviewing what style of starch in business starchnoodles is crucial to universal exchange, sanitation and defensive client benefit.

\section{Standardized Tag Scanner:}

The more seasoned sort is that the RS-232 standardized identification scanner. This thoughtful needs unique cryptography for moving the info document to the apparatus program. "Console interface scanners" report back to a pc utilizing a PS/2 or AT console adjusted association link. In any case, these plans aren't planned explicitly for the catching of standardized tags. Accordingly, they are doing not disentangle so rapidly or precisely as an over the top standardized identification scanner or helpful learning lethal.

\section{Serial CONVERTER:}

A USB connector might be a kind of convention gadget that is utilized for cleansing USB learning sign to and from elective interchanges standards. Ordinarily, USB connector's region unit won't to adjust USB learning to plain sequential marks information and a different way.

\section{VISA TOOL}

\section{Introduction TO VISA:}

The primary point of the VXI plug \& play Systems Association is to broaden straightforward use for completion clients through open, multi-seller procedures. The affiliation individuals share a standard prescience for multi-merchant procedures structure, together with every equipment and bundle. This normal prediction allows the individuals to figure along to diagram and power principles for framework level issues. As a stage toward assembling wide bundle similarity, the union created one style for $\mathrm{I} / \mathrm{O}$ programming - the Virtual Instrument System plan, or VISA. The VISA style characterizes a cutting edge I/O bundle standard not only for VXI, anyway conjointly for GPIB, Serial, and elective edges. VISA binds together the delivering to make bundle functional, recyclable, and ready to stand the check of your time. The affiliation conjointly arranged the principal across the board employable systems, application advancement certifications, and programming vernaculars into particular structures and sketched out top to bottom determinations to guarantee the capacity of modules inside each structure. 


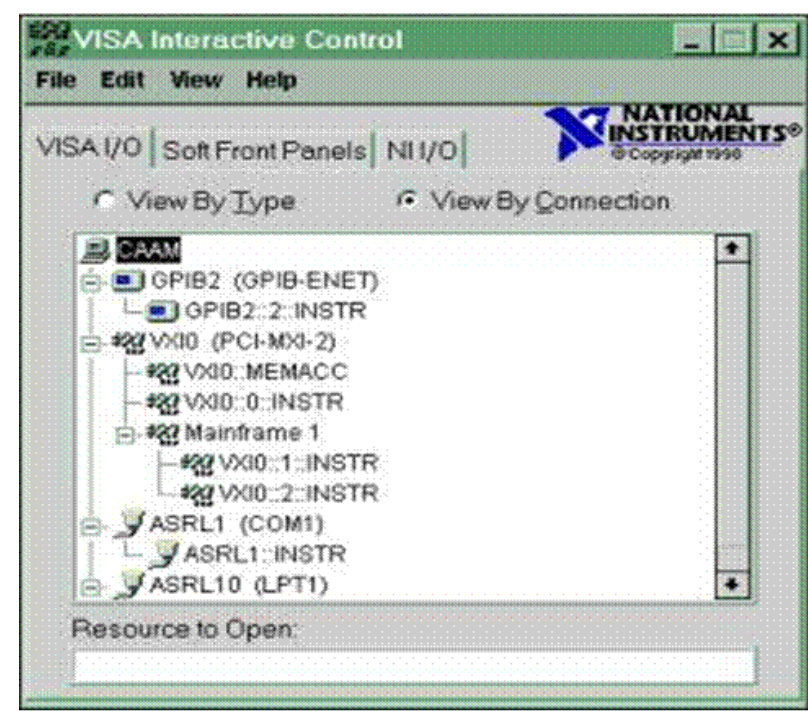

\section{LABVIEW TOOL}

\section{SERVER ARCHITECTURE}

The VI Server is AN article arranged, stage free innovation that gives automatic access to LabVIEW and LabVIEW applications. VI Server plays out a few capacities; however, this exercise focuses on exploitation the VI Server to control front board protests and alter the properties of a VI and LabVIEW. To realize an approach to utilize VI Server, it's useful to realize the terminology identified with it

\section{OBJECT ORIENTED TERMINOLOGY}

Item arranged writing computer programs is predicated on articles. an article might be an individual from a classification. A class characterizes what an article is in a situation to attempt to, what tasks it will perform (strategies), and what properties it's, similar to shading, estimate, etc. Articles will have ways and properties. Ways play out a task, such as reinitializing the thing to its default worth. Properties region unit the characteristics of an item. The properties of an item likely could be its size, shading, permeability, etc.

\section{CONTROL CLASSES}

LabVIEW front board items get properties and ways from a discourse. When you produce a Stop the board, it's an object of the Boolean class and has properties and ways identified with that classification.

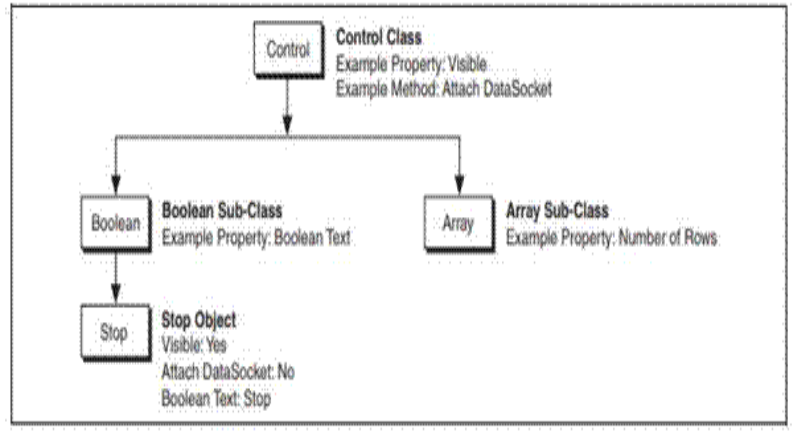

Boolean class example

\section{TESTING}

\section{Test Plan}

Programming animating is the technique for the examination that a product thing to acknowledge contrasts between given data and likely yield, and furthermore to assess the composition of a artefact thing. Analysis surveys the upside of the entry. Programming animating is a procedure that need to be finished during the development strategy. As such programming testing is a check and arrangement technique.

\section{Substantiation}

Confirmation is that the way to frame certain the product bear out the settings exacted en route for the start of the advancement segment. In elective words, to frame certain the product plays out the methodology we wish it to.

\section{Confirmation}

Endorsement is the strategy to safeguard the piece satisfies the predetermined necessities toward the end of the advancement stage. At the tip of the day, to confirm the item is worked in step with shopper wants.

\section{Making A SUBVI With Property Nodes}

The least complex approach to make expressly connected Property Nodes is to finish the accompanying advances:

1. Creation of VI is the initial step.

2. The standard of the square outline must be chosen which is in the sub VI, as like as the initial segment

3. Then go to Edit » Create Sub VI. Where the Lab VIEW naturally makes the control indications required for the sub VI.

4. Customize (if necessary) and spare the sub VI. As should be obvious in the second part, the sub VI utilizes the default symbol and connector piece. 


\section{OUTPUT \& RESULTS:}
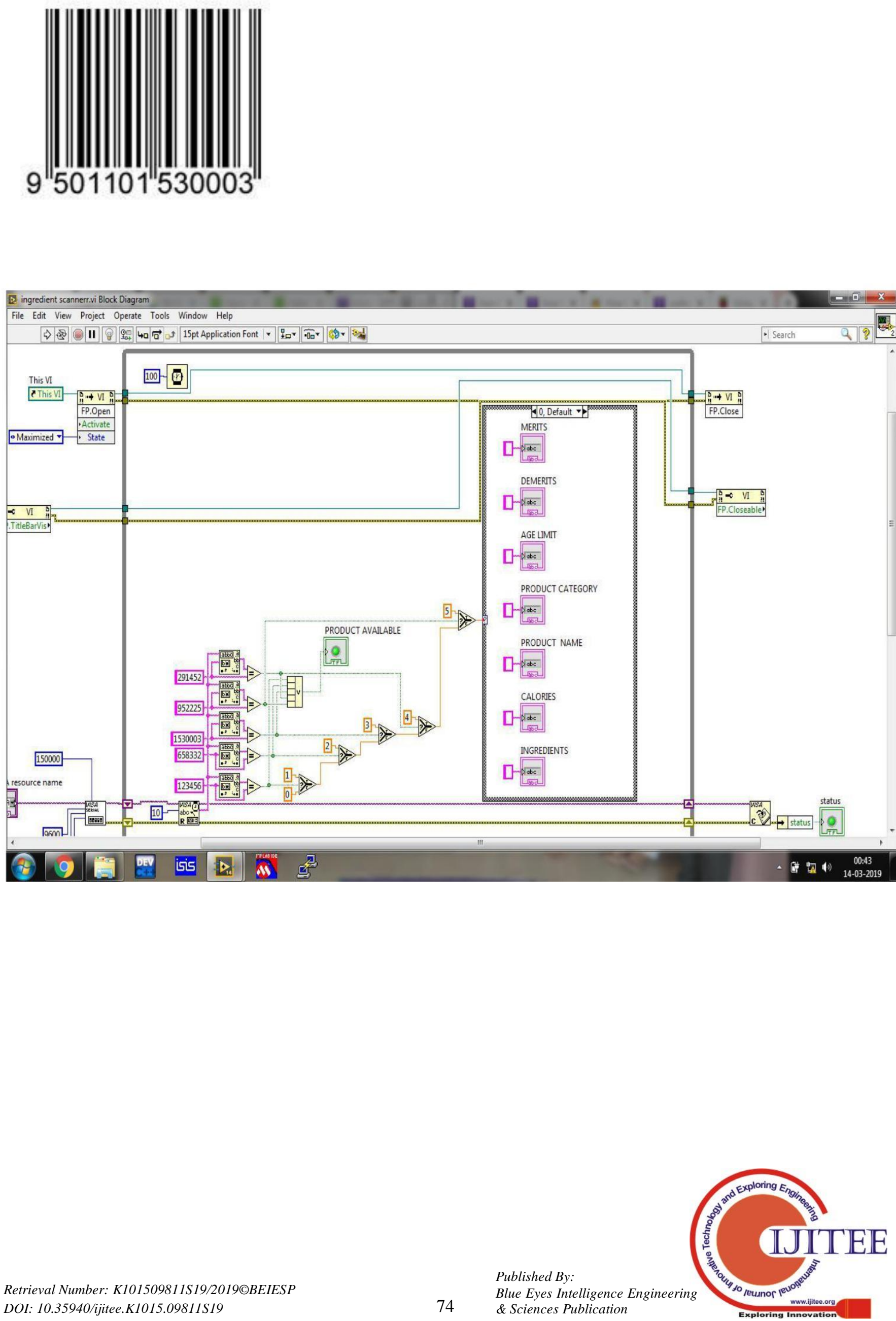
International Journal of Innovative Technology and Exploring Engineering (IJITEE) ISSN: 2278-3075, Volume-8, Issue-11S, September 2019

\section{$\||||||||||||||||||||| \mid$}
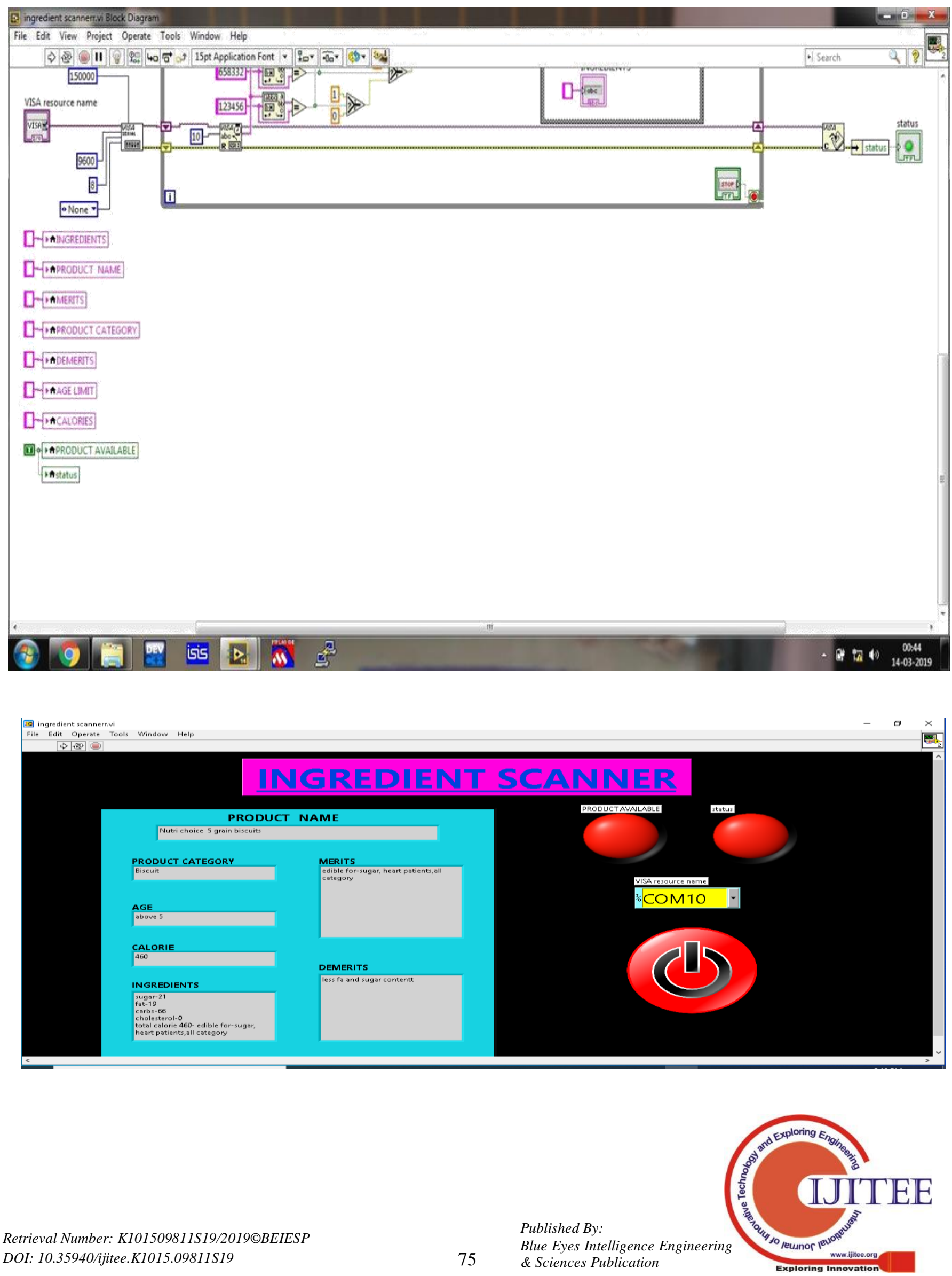


\section{CONCLUSION}

On further progress the application is developed as a mobile application in android and it will be easy for the customer to check the availability, merits and demerits of the product of what they are buying. This project is just an initiative and it can be further developed and updated in mobile phones and used by the customers. Hence limits are arranged into fixings utilizing LabVIEW dependent on the fixings level which is prudent for each age classes.

\section{REFERENCES}

1. Janssen et al., _Comparison of overweight and obesity prevalence in school-aged youth from 34 countries and their relationships with physical activity and dietary patterns, “' Obesity Rev., vol. 6, no. 2, pp. 123-132, 2005.

2. G. A. Bray and C. Bouchard, Handbook of Obesity: Clinical Applications, vol. 2. Boca Raton, FL, USA: CRC Press, 2014.

3. R. A. Robert and K. Len. Making Sense of CalorieBurning Claims. Accessed: Feb. 14, 2018. [Online]. Available: https://www.unm. edu/ lkravitz/Article\%20folder/caloricexp.html

4. M. Sun et al., _Determination of food portion size by image processing, " in Proc. 30th Annu. Int. Conf. IEEE Eng. Med. Biol. Soc. (EMBS), Aug. 2008, 871-874.

5. P. Pouladzadeh, S. Shirmohammadi, and A. Yassine, _Using graph cut segmentation for food calorie measurement,"“ in Proc. IEEE Int. Symp. Med. Meas. Appl. (MeMeA), Jun. 2014, pp. 1-6.

6. C. Nakayama et al., _Thermal-imaging of foods in heating process," in Proc. Int. Conf. Control, Autom. Syst. (ICCAS), Oct. 2008, pp. 950-953.

7. P. Pouladzadeh, G. Villalobos, R. Almaghrabi, and S. Shirmohammadi, A novel SVM based food recognition method for calorie measurement applications, " in Proc. IEEE Int. Conf. Multimedia Expo Workshops (ICMEW), Jul. 2012, pp. 495-498.

8. P. Pouladzadeh, S. Shirmohammadi, and T. Arici, Intelligent SVM based food intake measurement system, “ in Proc. IEEE Int. Conf. Comput. Intell. Virtual Environ. Meas. Syst. Appl. (CIVEMSA), Jul. 2013, pp. 87. 